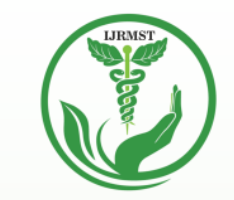

INTERNATIONAL JOURNAL OF RESEARCH IN MEDICAL SCIENCES \& TECHNOLOGY

e-ISSN:2455-5134; p-ISSN: 2455-9059

\title{
DEVELOPING AN INTEGRATED SYSTEM FOR THE CLINICAL SEGMENTATION OF LINER SCHEME CT IMAGES
}

\section{Armaan Jain}

Ryan International School, Rohini-25, New Delhi

Paper Received: 08 ${ }^{\text {th }}$ April, 2021; Paper Accepted: 07 ${ }^{\text {th }}$ May, 2021;

Paper Published: 29th June, 2021

DOI: http://doi.org/10.37648/ijrmst.vl li01.015

How to cite the article:

Armaan Jain, Developing an Integrated System for the Clinical Segmentation of Liner Scheme CT Images, IJRMST, January-June 2021, Vol 11, 156-166, DOI:

http://doi.org/10.37648/ijrmst.v11i01.015

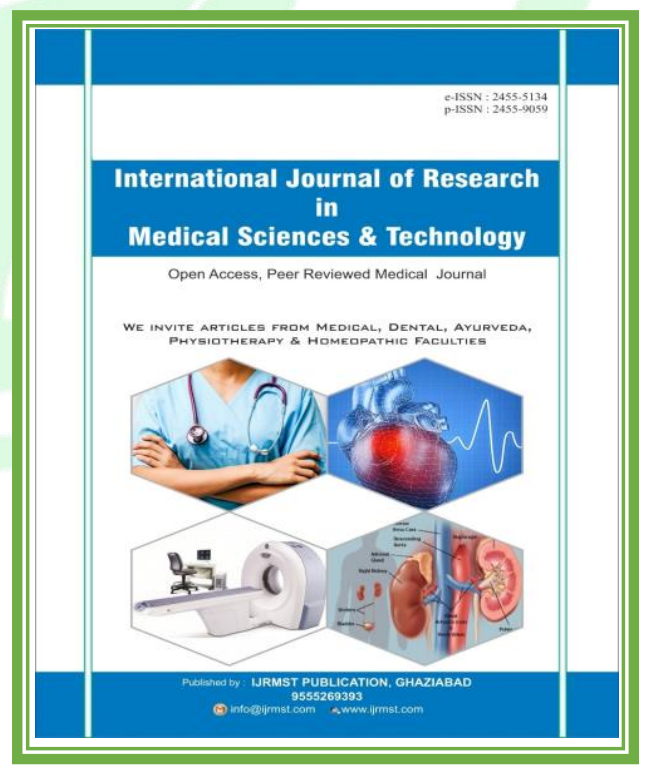




\section{ABSTRACT}

In clinical picture handling, the division of the liver in figured tomography pictures is of huge importance. To acquire liver segmentation, there is an examination of strategies for dividing the liver and methods utilizing processed tomography pictures. Isolating plans into two classes are self-loader and fully automated programs. The two classes have a few techniques, estimation, related questions; a few downsides will be depicted and explained. Following the similar review for liver division plans, different assessments and scoring are given; we will cautiously highlight the benefits and inconveniences of procedures. A few deficiencies and hardships of the proposed strategies are still focused around.

\section{INTRODUCTION}

These days, in clinical picture handling, the division of the liver in computed tomography pictures has tremendous significance. It is the beginning and significant activity for distinguishing liver infections, liver volume estimations and 3D liver volume delivery. A manual cycle and visual assessment are accustomed to drawing out the liver information or data, which is an exceptionally tedious interaction and process of thoughts to fix issues. Isolating the strategies for liver division into two classes is self-loader and methods of completely programmed division of the liver. The image preprocessing and AI hypotheses give more information about these two strategies for liver segmentation.

Moreover, it isn't simple regarding the low-level difference and unclear limits used to recognize the figured tomography pictures. The incomplete volume impacts produce the above highlights due to spatial averaging, patient development, and shaft solidifying. Likewise, a few shaded levels might be utilized by adjoining organs in the body like the spleen, liver, and stomach. Similar dark levels can't use a similar organ identified with a similar theme. This multitude of attributes, the problem with the tremendous variety of liver shapes, upgrade the issue in the liver segmentation study.

\section{LIVER VOLUME SEGMENTS}

The liver division by $\mathrm{CT}$ pictures is separated into two distinct classes: fractional/self-loader strategy and programmed liver division technique. 


\section{VOLUME: 1 1, JANUARY-JUNE 2021}

\section{Segmentation of Liver Using Semi-}

\section{Automatic Schemes}

1. The most recent Semi-programmed strategies for division of the liver are reachable, and as indicated by picture pre-processing, these techniques are foreordained. These plans need a minor user contribution, which is utilized to get done with the job. The interest for this undertaking is changed from picked of seed pixels physically to a manual refinement of a twofold cover for the liver.

The inclusion of client utilized in the strategies for division of liver through the activity for the choice of seed focuses and using steps for change.

Graph-based self-loader plans Images are taken care of by weighted and undirected diagrams, where pixels are known as the vertices, and adjoining pixels are considered associated vertices. Loads of the edges in the diagram ascertain the resemblance among two comparing vertices. Typically, the live wire calculations and the diagram cut division calculations are utilized under this class.

Barrett and Mortensen (1997) arranged a calculation for live wire division to eliminate edges in clinical pictures. Observing the smallest expense ways among seed focuses that the client now determines by analyzing live wire division. The weighted amount of Image elements, for example, the slope esteem, dark worth, inclination bearing, and Laplacian filtering intersection, are utilized to figure the way's expense. Right off the bat, the client will choose the underlying seed point, which lies on the limit of the organ, later that the opening from the seed point is selected (currently ordered by the client in the picture). Dijkstra's search calculation or dynamic programming calculations are utilized to compute all conceivable smallest expense ways. The client will choose the limit of the image.

Schenk et al. (2001) extend the above liver-wire strategy for liver division in computed tomography pictures, which likewise assists with diminishing the client's correspondence and computation period. The expense work is determined by deciding the liver shape from the closest neighbouring cut in the body, which is portioned now. The client can deal with the division course upheld by the liverwire division computation. The client's work will be confined by picking the seed focuses and choosing the most needed edges, while the processor will deal with the subtleties. 


\section{VOLUME: 11 , JANUARY-JUNE 2021}

Beichel et al. (2007) utilized the graph cut division calculation in their exploration. They expected computed tomography pictures relying upon the strategy for graph cut division and the 3D intuitive liver division approach.

2. Region-developing based self-loader plans

This method depends on reality in which the normal dark qualities are shared by close pixels. For the most part, this technique is utilized in an iterative or replication way where the entire organ is fragmented inside the liver in particular regions. Physically late pixels are added to the seed region as the force of an encompassing region is beneath that of seed power under a given restricted worth. Beck and Aurich (2007) occupied their methodology area developing calculation of cooperation liver division. They expected three-aspect district producers through the nonlinear coupling rule. The client physically amends the spilt districts or missing parts by working out the arched frame inside limited neighbourhoods the limit, the division continues. This interaction is known as the post-handling step.

3. Level sets based self-loader plans

In this strategy, the client delineates a short form from inside or outside the item, and afterwards, the shape will contract/augment. This calculation goes under the picture division issue. Will end the most common way of gaining/expanding when the body meets the item limit. The significant capacity dealing with the method of form contracting/extending additionally deciding the endpoint of this cycle is finished by speed work. Liver division techniques under self-loader are ordered into two gatherings, which are 2D level sets systems, and 3D level creates methodologies.

\section{Atlas matching self-loader plans}

Probabilistic map books are set up from numerous physical pictures by manual division. By utilizing relative changes, images have been submitted into a typical space. These pictures and comparing divisions are then found the median value of and connected with a Bayesian edge for developing a probabilistic Atlas; for each pixel, the haphazardness for a specific organ is determined. Finally, basic thresholding or restrictive mode calculation is utilized to draw out the vital organ, relying on the later likelihood. The probabilistic Atlas requires a ton of preparing information that can be assembled and divided, its principal 
impediment.

\section{Fully-Programmed Liver Division Plans}

By - fully automatedll, we imply that we will apply the division cycle of the liver with practically no client contribution. Regularly, completely programmed liver division techniques are profoundly esteemed by radiologists and delivered by udders issues and favouritism. It is troublesome and a wastage of time and saves the administrator from these disadvantages.

\section{Rule-based programmed techniques}

Chi et al. (2007) utilize committed prearranging language: portray conventions used to eliminate the various associations from the generally tried pictures. The extraction request is foundation air, lungs and other intrabody air, subcutaneous fat and muscle layer, bones inside muscle layer, aorta, spine, heart, and liver. Picture investigation is done when an association utilizes the recently identified design during every expulsion step. These conventions can likewise incorporate neighbourhood relations, power conveyances, mathematical highlights, and so forth. A seed space is chosen by edge the right half of the CT cut beneath the heart after containing the eliminated recorded association up to the seat until a thing adjusting certain size standards is seen. A cycle identified with locale development is started by utilizing this seed area. All guidelines are characterized without really any utilization of provided preparing data and elements that have not been methodically assessed.

\section{Gray-level based programmed plans}

Dark level put together programmed strategies to depend concerning factual investigation for figured tomography portions that are sectioned to compute the dim liver levels. A few tasks use histogram examination that relies on past information regarding the liver force range for ascertaining the dark liver levels. The determined qualities are utilized with a clear or cyclic thresholding interaction to build a twofold guide that describes the liver. Then, at that point, this picture is handled morphologically to eliminate associated organs. The current sectioned image gives the data to help divide the current epitome of embodiment. Finally, if it's not too much trouble, using dynamic shapes or B spines helps with smoothing the edges of each processed tomography pictures

Website optimization and Park (2005) proposed a plan for the liver division interestingly improved registered 


\section{VOLUME: 1 1, JANUARY-JUNE 2021}

tomography pictures, which rely on calculating left fractional histogram edge (LPHT). The left halfway histogram limit eliminates other adjoining organs from the pixels varieties. A multi-modular section follows histogram changes used to track down the

scopes of dark levels. Ultimately, morphological sifting is figuring out how to smooth the picture's edges and eliminate undesirable things.

The extraction of the liver in figured tomography pictures and utilized in PC supported liver investigation framework; this plan is arranged by Pil et al. (2006). Estimated the liver dispersion, likewise used to pick the locale of interest. When the likelihood crosses half, the window will be distributed as a locale of interest contrasted with the liver's benefit of existing probability. Then, at that point, the watershed division calculation is utilized to mine the liver locales. Can join the divided regions into the ground breaking areas used for ideal division. Ultimately, the size of the liver is picked by past information in regards to the anatomic data of the liver.

\section{RELATED STUDIES}

The procedures for the liver shape model over and again come up short. When there is a complex formed liver, the techniques

for the liver shape model consistently come up short. Different structures additionally go through the normal issue; they couldn't separate the organs connected to the liver. In registered tomography cuts, the associated organs have a comparative power outside. Consequently, discontinuity of the liver, inconveniences are still there. The various methods are not significant because of the requirement for normal information and a particular computation. Additionally, unmistakable styles and techniques are capable of little informational indexes. Further, strategies execution is determined, which relies upon self-chose issue capacities.

Heimann et al. (2009) determined sixteen programmed and intelligent plans for the liver division. A lot bigger standard deviation of the closure scores can be inspected via robotized strategies when connecting to the natural and the imaginative segmentation draws near. The enormous standard deviation happens because of the shortcomings structure of anomalies. Albeit the examination of programmed mode and intuitive have similar a few effective results in the correlation investigation of intelligent procedures, ordinarily, the consistency of mechanized cycles is more unfortunate. 


\section{VOLUME: 11 , JANUARY-JUNE 2021}

Inconveniences happen at detailed test pictures and regions. Even though few areas cause more issues than different districts, all techniques fall flat, not even in a solitary part. While assessing execution, this perception, along with the extraordinary assortment of results over various test pictures, upholds the required huge and different variety of tests. With the precision of the made result, techniques are determined. Will notice division with extraordinary exactness.

\section{CONCLUSION}

The two plans for a liver division are selfloader and programmed liver division, utilizing processed tomography pictures related strategies and ideas, have been analysed. Even though numerous techniques for division are tried, inconveniences consistently lie there. In assessed ways, the degree of dim-based strategies is utilized to accomplish the ideal outcomes, yet they are not that solid for data set variety. The high inconstancy of CT force esteems doesn't inspect by dark level assessment. The presentation could diminish altogether when complex and huge informational indexes are utilized.

What's more, a few techniques need actual inclusions and some natural boundaries to be tentatively assessed. This large number of realities impacts the vigour strategy. Learning strategies depend on the preparation set and ought to choose watchfully. There is a necessity for a lot of data can be precisely accumulated, likewise can be truly divided to make the structure, the model-based procedures, and probabilistic map books go through some trouble; this is because the preparation set, just as deficiencies of clients and shamefulness, are solid influences the got model. Beginning task changes the result of the division. The calculations will be fruitless while managing non-standard liver shapes. It isn't easy to portray a legitimate speed capacity, and its elements are the primary limit. Moreover, a connection between the various strategies isn't significant due to the requirement for normal information and exact computation. Moreover, utilized datasets in general examiners are incredibly not many. 


\section{VOLUME: 11 , JANUARY-JUNE 2021}

\section{REFERENCES}

[1]. Arya S, Mount DM, Netanyahu NS, Silverman R, Wu A (1998) an optimal algorithm for approximate nearest neighbor searching. J ACM 45(6):891-923

[2]. Barrett W, Mortensen EN (1997) Interactive live-wire boundary extraction. Med Imaging Anal 1(4):331-341

[3]. Beck A, Aurich V (2007) HepaTux-a semiautomatic liver segmentation system. In: Proceedings of MICCAI Workshop on 3D segmentation in the clinic: a grand challenge pp225-234

[4]. Beichel R, Bauer C, Bornik A, Sorantin E, Bischof H (2007) Liver segmentation in CT data: a segmentation refinement approach. In: Proceedings of MICCAI workshop on 3D segmentation in the clinic: a grand Challenge, pp 235-245

[5]. Campadelli P, Casiraghi E, Esposito A (2009) Liver segmentation from computed tomography scans: a survey and a new algorithm. Artif Intell Med 45(2-3):185-196

[6]. Carr JC, Beatson RK, Cherrie JB, Mitchell TJ, Fright WR, McCallum BC, Evans TR (2001) Reconstruction and representation of 3-D objects with radial basis functions. In: Proceedings of SIGGRAPH, pp 67-76

[7]. Chi Y, Cashman PMM, Bello F, Kitney RI,(2007) A discussion on the evaluation of a new automatic liver volume segmentation method for specified CT image datasets. In: Proceedings of MICCAI workshop on 3D segmentation in the clinic: a grand challenge, pp $167-175$

[8]. Cootes TF, Hill A, Taylor CJ, Haslam J (1994) Use of active shape models for locating structures in medical images. Imag Vis Comput 12(6):355-366

[9]. Heimann $\mathrm{T}$ et al (2009) Comparison and evaluation of methods for liver segmentation from CT datasets. IEEE Trans Med Imaging 28(8):1251-1265

[10]. Kainmüller D, Lange T, Lamecker H (2007) Shape constrained automatic segmentation of the liver based on a heuristic intensity model. In: Proceedings of MICCAI workshop on 3D segmentation in the clinic: a grand challenge, pp 109-116 


\section{VOLUME: 11 , JANUARY-JUNE 2021}

[11]. Koss JE,Newman FD, Johnson TK,Kirch DL (1999) Abdominal organ segmentation using texture transforms and a hopfield neural network. IEEE Trans Med Imaging 18(7):640648

[12]. Lamecker H, Lange R, SeebaM (2004) Segmentation of the liver using a 3d statistical shape model. Technical report Zuse Institue, Berlin, pp 1-25

[13]. Lee CC, Chung PC, Tsa H (2003) Identifying multiple abdominal organs from CT image series using a multimodule contextual neural network and spatial fuzzy rules. IEEE Trans Inf Technol Biomed 7(3):208-217

[14]. Lim SJ, Jeong, YY, Ho YS (2004) Automatic segmentation of the liver in ct images using the watershed algorithm based on morphological filtering. In: Proceedings of SPIE, pp $1658-1666$

[15]. Lim SJ, Jeong, YY, Ho YS (2005) Segmentation of the liver using the deformable contour method on CT images. In: Proceedings of SPIE medical imaging, pp 570-581

[16]. Lim SJ, Jeong YY, Ho YS (2006) Automatic liver segmentation for volume measurement in CT Images. JVCIR 17(4):860-875

[17]. Mattes D, Haynor DR, Vesselle H, Lewellen TK, Eubank W (2003) PET-CT image registration in the chest using free-form deformations. IEEE Trans Med Imaging 22(1):120128

[18]. Montagnat J, Delingette H (1996) Volumetric medical images segmentation using shape constrained deformable models. In: Proceedings of CVRMed-MRCAS, pp 13-22

[19]. Pil UK, Yun JL, Youngjin J, Jin HC, Myoung NK, (2006) Liver extraction in the abdominal CT image by watershed segmentation algorithm. World congress of medical physics and biomedical engineering, pp 2563-2566

[20]. Rikxoort E, Arzhaeva Y, Ginneken B (2007) Automatic segmentation of the liver in computed tomography scans with voxel classification and atlas matching. In: Proceedings of MICCAI workshop on 3D segmentation in the clinic: a grand challenge, pp 101-108

[21]. Rohlfing T, Brandt R, Menzel R, Russakoff DB, Maurer CR (2005) Quo vadis, atlas based segmentation? 


\section{VOLUME: 1 1, JANUARY-JUNE 2021}

[22]. Handbook of medical image analysis-Volume III: Registration models. Kluwer Academic, Norwell MA, pp 435-486

[23]. Rousson M, Cremers D (2005) Efficient kernel density estimation of shape and intensity priors for level set segmentation. In: Proceedings of MICCAI, pp 757-764

[24]. RueckertD, Sonoda LI,Hayes C, Hill DL,LeachMO,HawkesDJ (1999) Nonrigid registration using free-form deformations: application to breast MR images. IEEE Trans Med Imaging 18(8):712-721

[25]. Schenk A, Prause GP, Peitgen H (2001) Local cost computation for efficient segmentation of $3 \mathrm{~d}$ objects with live wire. In: Proceedings of SPIE on medical imaging, pp $1357-1364$

[26]. Seo KS, Park JA (2005) Improved automatic liver segmentation of a contrast enhanced CT image. Advances in multimedia information process_-PCM, pp 899-909

[27]. Slagmolen P, Elen A, Seghers D, Loeckx D, Maes F, Haustermans, K (2007) Atlas based liver segmentation using no rigid registration with a B-spline transformation model. In: Proceedings of MICCAI workshop on 3D segmentation in the clinic: a grand challenge, pp 197-206

[28]. Soler L, Delingette H, Malandain G, Montagnat J, Ayache N, Koehl C, Dourthe O, Malassagne B, Smith M,

[29]. Mutter D, Marescaux J (2001) Fully automatic anatomical, pathological, and functional segmentation from ct scans for hepatic surgery. Comput Aided Surg 6(3):131-142

[30]. Sonka M, Hlavac V, Boyle R (2007) Mathematical morphology in image processing, analysis, and machine vision. Thomson, Newyork

[31]. Susomboon R, Raicu DS, Furst J (2007) Ahybrid approach for liver segmentation. In: Proceedings of MICCAI Workshop on 3D

segmentation in the clinic: a grand challenge, pp 151-160

[32]. Tsai D, Tanahashi N (1994) Neural-network-based boundary detection of liver structure in ct images for 3-d visualization. In: Proceedings of IEEE international conference neural networks, pp 3484-3489 


\section{VOLUME: 1 1, JANUARY-JUNE 2021}

[33]. Tsai A, Yezzi A, Wells W, Tempany C, Tucker D, Fan A, Grimson W, Willsky A (2003) A shape- based approach to the segmentation of medical imagery using level sets. IEEE TransMed Imaging 22(2):137-154

[34]. Weickert J, Romeny BMTH, Viergever MA (1998) Efficient and reliable schemes for nonlinear diffusion filtering. IEEE Trans Imaging Process 7(3):398-410

[35]. Wimmer A, Soza G, Hornegger J (2007) Two-stage semi-automatic organ segmentation framework using radial basis functions and level sets: In: Proceedings of MICCAI workshop on 3D segmentation in the clinic: a grand challenge, pp 179-188 